\title{
Citogenética de Calochortus barbatus (Liliaceae: Calochortoideae) de la Reserva Ecológica del Pedregal de San Ángel, en México, Distrito Federal
}

\author{
Cytogenetics of Calochortus barbatus (Liliaceae: Calochortoideae) of the Reserva Ecológica del \\ Pedregal de San Ángel, Distrito Federal, Mexico
}

\author{
Fernando Tapia-Pastrana*
}

Laboratorio de Genecología, División de Estudios de Posgrado e Investigación, Facultad de Estudios Superiores Zaragoza, Universidad Nacional Autónoma de México, Apartado postal 9-020, 15000, México, D.F.

*Correspondencia: pasfer@correo.unam.mx

\begin{abstract}
Resumen. Se realizó un estudio citogenético en células de meristemos radiculares de individuos de Calochortus barbatus de la Reserva Ecológica del Pedregal de San Ángel, en México, D.F. Luego de aplicar una metodología de extendido y secado al aire se confirma un número cromosómico $2 n=4 x=36(x=9)$ y se propone por vez primera una fórmula cariotípica para la especie $(14 \mathrm{~m}+22 \mathrm{st})$. El cariotipo asimétrico (T.F. $\%=23.13)$, que incluye 4 cromosomas subtelocéntricos pequeños portadores de constricciones secundarias y satélites, permite descartar la presencia de fragmentos. La longitud cromosómica total haploide $(53.48 \mu \mathrm{m})$ y el intervalo cromosómico $(1.85-4.27 \mu \mathrm{m})$ añaden datos novedosos sobre la arquitectura cromosómica. Se discute el papel de la hibridación intraespecífica y autoploidía en la conformación del cariotipo de esta especie y la necesidad de realizar estudios similares en especies estrechamente relacionadas.
\end{abstract}

Palabras clave: autoploidía, Calochortus, extendido y secado al aire, cariotipo, satélites, hibridación intraespecífica.

\begin{abstract}
Meristematic root cells from seeds of individuals of Calochortus barbatus from Reserva Ecológica del Pedregal de San Ángel, Distrito Federal, Mexico were analyzed cytogenetically using a surface spreading and airdrying method. The chromosomal number, $2 n=4 x=36(x=9)$ was corroborated. The mitotic karyotype was established for the first time for this species as $14 \mathrm{~m}+22 \mathrm{st}$. The asymmetric karyotype, which includes 4 small subtelocentric chromosomes bearing a secondary constriction with its corresponding satellites, allows to discard previous reports of chromosomal fragments. Total haploid chromosomal length $(53.48 \mu \mathrm{m})$, chromosomal length range $(1.85-4.27 \mu \mathrm{m})$ and the asymmetry index (T.F. $\%=23.13$ ) add new information on chromosome architecture. The role of intraspecific hybridization and autoploidy in shaping the karyotype of this species and the need of performing similar analyses in related species are discussed.
\end{abstract}

Key words: autoploidy, Calochortus, spreading and air-drying, karyotype, satellites, intraspecific hibridization.

\section{Introducción}

Calochortus incluye 67 especies y algunos híbridos naturales, subespecies y variedades de geófitas bulbosas, cuyo centro de diversidad es California, Estados Unidos de América (EUA). Este género tiene una distribución desde el norte de Columbia Británica, Canadá a través del oeste de las Dakotas, EUA hasta México y Guatemala (Ownbey, 1940; Nesom, 1983; Patterson y Givnish, 2003). La sistemática del género es difícil y la determinación y arreglo taxonómico de muchas de sus especies quedan por resolver (Beal, 1939). Hasta el momento las especies se incluyen

Recibido: 21 abril 2009; aceptado: 06 febrero 2010 en 3 secciones y 12 subsecciones propuestas por Ownbey (1940). Asimismo, debido a la rareza y a la distribución extremadamente restringida de algunas de sus especies, se ha planteado que se realicen esfuerzos para su inmediata conservación (Fredricks, 1989).

Calochortus barbatus (Kunth) J.H. Painter pertenece a la sección Cyclobothra, subsección Barbati (Ownbey, 1940; Beal y Ownbey, 1943) y se ubica en el clado denominado México Central en la filogenia del género propuesta por Patterson y Givnish (2003). Es una especie variable, común en pastizales, bosques de pino-encino, orillas de camino y lugares perturbados. En Norteamérica presenta un amplio intervalo de distribución, desde el SO de Estados Unidos hasta los estados del altiplano en México, desde Chihuahua hasta Oaxaca, donde crece predomi- 
nantemente en suelos volcánicos (Ownbey, 1940). En el Valle de México se le conoce comúnmente como ayatito (Martínez, 1994) y tiene una distribución altitudinal entre 2200 y $3100 \mathrm{~m}$ snm. Es claramente reconocible por sus flores amarillas, amarillo-rojizas o amarillo-purpúreas con pétalos densamente barbados, frutos secos lineares y semillas comprimidas. Florece y fructifica de junio a diciembre (Ownbey, 1940; McVaugh, 1989; Rzedowski y Rzedowski, 2001).

Los estudios previos sobre la citogenética de las especies de Calochortus muestran una estrecha correlación entre el número cromosómico y la posición sistemática, con una clara separación de sus 3 secciones según el número básico: sección Calochortus $(x=10)$; sección Mariposa ( $x$ $=6,7,8,9)$ y sección Cyclobothra $(x=9)$ (Beal, 1939; Beal y Ownbey, 1943; Cave, 1970). Estos estudios, realizados a partir de metodologías de cortes transversales hoy día en desuso, describen una notoria similitud de tamaño y morfología entre los cromosomas de las especies asignadas a tales secciones, así como la ocurrencia de ciertas formas en común en todas las especies (Newton, 1926; Beal, 1939).

En la presente investigación se realizó un estudio citogenético utilizando una metodología de extendido y secado al aire para caracterizar la arquitectura de los cromosomas somáticos de una muestra de individuos de C. barbatus de la Reserva Ecológica del Pedregal de San Ángel en México, Distrito Federal, éste es el único taxón tetraploide de la sección Cyclobothra $(2 n=4 x=36, x=9)$ (Beal, 1941; Beal y Ownbey, 1943). La obtención de la fórmula cromosómica, el cariotipo y otros datos citológicos son de interés para comprender aspectos básicos del sistema genético y la evolución cromosómica de esta especie, cuya distribución en nuestro país abarca una gran diversidad de ambientes.

\section{Materiales y métodos}

Se recolectaron frutos de C. barbatus en 2 áreas de amortiguamiento de la Reserva Ecológica del Pedregal de San Ángel de Ciudad Universitaria: zona A5 (Paseo de las Esculturas) y zona A10 (Jardín Botánico), en el sur de la cuenca del Valle de México (entre 19 20' 2" y $19^{\circ} 13^{\prime} 45^{\prime}$ "

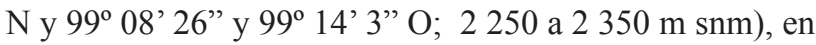
el mes de octubre de 2007. Se seleccionaron al azar semillas provenientes de al menos 8 individuos de cada zona y fueron puestas a germinar a temperatura ambiente y en luz natural en cajas de Petri con algodón humedecido con agua destilada. Las raíces de 1-2 cm de largo fueron cortadas y pretratadas en 8-hidroxiquinoleína $0.002 \mathrm{M}$ durante 5 horas a temperatura ambiente. Posteriormente fueron fija- das en solución Farmer (etanol-ácido acético, 3:1). Para la obtención de los cromosomas metafásicos se siguió el método de secado al aire (Tapia-Pastrana y MercadoRuaro, 2001), el cual se basa en la maceración enzimática (pectinasa $20 \%+$ celulasa $2 \%$ ) de los meristemos apicales durante 2 horas a $37^{\circ} \mathrm{C}$, seguido de la transferencia del botón celular a una solución de $\mathrm{KCl} 0.075 \mathrm{M}$ durante 20 minutos a $37^{\circ} \mathrm{C}$. A continuación se realizaron 2 lavados con la misma solución y el botón celular se fijó en solución Farmer. Las laminillas se prepararon dejando caer 2 gotas del botón celular sobre el portaobjetos. Los cromosomas se tiñieron con Giemsa al 10\% y las preparaciones se hicieron permanentes empleando Entellán Nuevo (Merck) como medio de montaje. En un microscopio óptico Zeiss Axioscop, con película Kodak Technical Pan se fotografiaron los mejores campos.

Se utilizó un vernier digital (Mitutoyo Digimatic Caliber CD-G'BS) para establecer las tallas cromosómicas sobre 6 fotografías de placas en metafase típica con la misma magnificación. Para la obtención de la fórmula cariotípica se aplicó el sistema propuesto por Levan et al. (1964). La proporción de la suma total de longitudes de brazos cortos respecto a la suma total de longitudes cromosómicas como indicador de simetría o asimetría de un cariotipo (T.F.\%) se realizó según Sinha y Roy (1979). Con la finalidad de descartar la presencia de fragmentos cromosómicos o cromosomas $\mathrm{B}$, también se analizaron núcleos en anafase.

\section{Resultados}

Se revisaron 123 núcleos en metafase que mostraron un número cromosómico diploide $2 n=4 x=36,(x=9)$; los cromosomas de tamaño relativamente grande con un intervalo de 1.85 a $4.27 \mu \mathrm{m}$ exhibieron una longitud cromosómica total haploide (LCTH) de $53.48 \mu \mathrm{m}$ (Cuadro 1) y un índice de simetría (T. F.\%) de 23.13, que indica un cariotipo asimétrico. El cariotipo de los individuos de C. barbatus bajo estudio incluye 14 cromosomas submetacéntricos y 22 cromosomas subtelocéntricos $(14 \mathrm{sm}+$ $22 \mathrm{st}$ ), con 2 pares de cromosomas subtelocéntricos (uno heteromórfico) que exhiben constricciones secundarias y portan satélites cuyo tamaño varía en relación al grado de contracción de los cromosomas en metafase (Figs. 1 y 2). El análisis de núcleos en anafase no reveló la presencia de fragmentos cromosómicos. 
Cuadro 1. Medidas cromosómicas promedio en Calochortus barbatus obtenidas de 6 células en metafase típica ${ }^{1}$

\begin{tabular}{|c|c|c|c|c|c|}
\hline$P C$ & $L C$ & $L B L$ & $L B C$ & $r$ & $N$ \\
\hline 1 & 4.27 & 2.89 & 1.36 & 2.12 & $\mathrm{sm}$ \\
\hline 2 & 3.81 & 2.78 & 1.04 & 2.67 & $\mathrm{sm}$ \\
\hline 3 & 3.81 & 3.09 & 0.71 & 4.35 & st \\
\hline 4 & 3.47 & 2.80 & 0.66 & 4.24 & st \\
\hline 5 & 3.27 & 2.33 & 0.94 & 2.47 & $\mathrm{sm}$ \\
\hline 6 & 3.25 & 2.68 & 0.51 & 5.25 & st \\
\hline 7 & 3.17 & 2.48 & 0.68 & 3.64 & st \\
\hline 8 & 3.05 & 2.37 & 0.66 & 3.59 & st \\
\hline 9 & 3.01 & 2.12 & 0.88 & 2.40 & $\mathrm{sm}$ \\
\hline 10 & 2.99 & 2.24 & 0.61 & 3.67 & st \\
\hline 11 & 2.90 & 2.38 & 0.52 & 4.57 & st \\
\hline 12 & 2.72 & 1.91 & 0.81 & 2.35 & $\mathrm{sm}$ \\
\hline 13 & 2.69 & 2.22 & 0.46 & 4.82 & st \\
\hline 14 & 2.48 & 2.01 & 0.46 & 4.36 & st \\
\hline 15 & 2.36 & 1.67 & 0.68 & 2.45 & $\mathrm{sm}$ \\
\hline 16 & 2.25 & 1.78 & 0.46 & 3.86 & $\mathrm{st}^{*}$ \\
\hline 17 & 2.13 & 1.50 & 0.61 & 2.45 & $\mathrm{sm}$ \\
\hline 18 & 1.85 & 1.51 & 0.32 & 4.71 & $\mathrm{st}^{*}$ \\
\hline
\end{tabular}

${ }^{1}$ Abreviaturas: $P C$, par cromosómico; $L C$,longitud cromosómica en $\mu \mathrm{m} ; L B L$, longitud brazo largo en $\mu \mathrm{m} ; L B C$, longitud brazo corto en $\mu \mathrm{m} ; r$, proporción de brazos; $N$, nomenclatura con base en Levan et al. (1964). *, Cromosoma con constricción secundaria y satélites; sm, submetacéntrico; st, subtelocéntrico.

\section{Discusión}

En el único estudio previo que incluye datos sobre la citogenética de C. barbatus, Beal (1941) registró un número cromosómico $2 n=36$ y cromosomas que en algunos casos aparecían en cuadruplicado, por lo que la condición tetraploide $(x=9,4 x=36)$ quedó establecida. Sobre la morfología cromosómica, no obstante, sólo se mostró el dibujo de un complemento cromosómico obtenido a partir de cortes transversales de meristemos embebidos en parafina. Por otra parte, Cave (1970) señaló que en las secciones de Calochortus, tanto los complementos diploides como los tetraploides, exhiben respectivamente 1 o 2 pares de cromosomas ligeramente mayores que los restantes y que los cromosomas, particularmente en la condición tetraploide, no eran fácilmente identificables.

Los resultados obtenidos en esta investigación confirman en $C$. barbatus un número cromosómico $2 n=4 x=$ 36 , acorde con la propuesta de $x=9$ para la sección $C y c$ lobothra, así como un complemento cromosómico donde predominan los cromosomas con centrómero desplazado (Figs. 1 y 2). Sin embargo, el cariotipo de los individuos de $C$. barbatus aquí analizados muestra que los cromosomas disminuyen gradualmente su talla, en tanto que la identificación individual de los cromosomas se realiza con 
relativa facilidad. Lo anterior queda de manifiesto al adelantar la primera propuesta de fórmula cariotípica para una población de esta especie $(14 \mathrm{sm}+22 \mathrm{st})$, la cual se caracteriza por incluir 2 pares de cromosomas subtelocéntricos (st) portadores de constricción secundaria y con satélites evidentes (Fig. 2 y Cuadro1).

Hasta ahora, la presencia de satélites en especies del género Calochortus no había sido confirmada, pues sólo se registraron descripciones ambiguas, tales como cromosomas con constricciones similares a una condición satelital en $C$. albus Dougl. ex Benth. (sección Calochortus); la

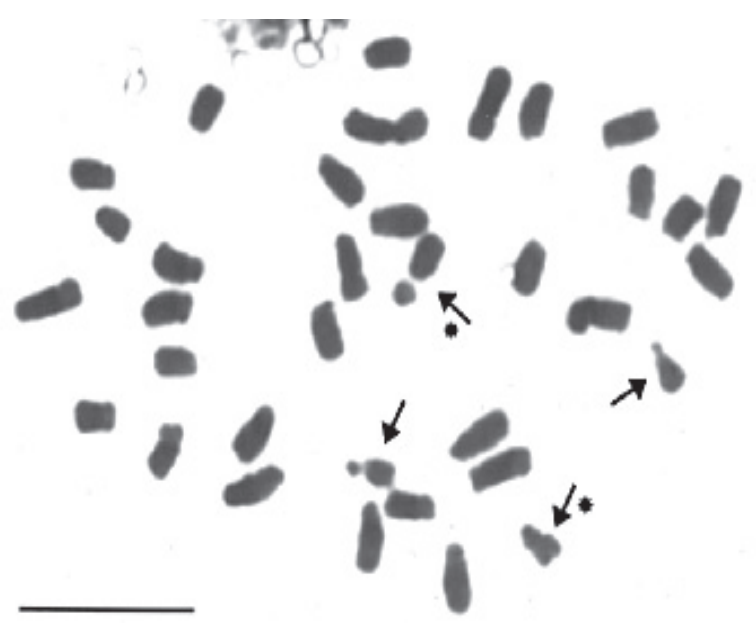

Figura 1. Metafase mitótica de Calochortus barbatus $(2 \mathrm{n}=4 \mathrm{x}=$ 36). Las flechas señalan los cromosomas con constricciones secundarias y porción satélite. *Par heteromórfico. Escala $=10 \mu \mathrm{m}$. posibilidad de que $C$. catalinae S. Wats. (sección Mariposa) exhiba satélites, o bien, la presencia de fragmentos semejantes a satélites adheridos lateral o terminalmente a cromosomas de C. venustus Dougl. ex Benth. (Beal, 1939).

La presente investigación revela que en los complementos cromosómicos de $C$. barbatus tanto las constricciones secundarias como los satélites se encuentran invariablemente asociados a los 4 cromosomas subtelocéntricos (st) más pequeños (Figs. 1 y 2). Cabe señalar que algunas constricciones separan notablemente los satélites y aunque este evento es común en especies vegetales (Tapia-Pastrana et al., 2005; Tapia-Pastrana, 2007), podría ocasionar que éstos sean confundidos con fragmentos cromosómicos, más aún cuando se trata de satélites grandes como los presentes en C. barbatus. Esto podría explicar algunas descripciones anteriores sobre la presencia de fragmentos en células de individuos que por lo demás en nada difieren de aquellos en los que no fueron observados (Beal, 1939).

En relación con la presencia de cromosomas homólogos en cuadruplicado, esta investigación muestra que tal condición es verificable particularmente en los primeros 4 pares del cariotipo de C. barbatus (Fig. 2). Sin embargo, también es claro que no se ajusta a todos los cromosomas del complemento, particularmente en el par 16 (heteromórfico) cuya talla, tamaño del satélite y relación de brazos son notoriamente diferentes (Cuadro 1). En opinión de algunos autores, la ausencia de duplicación perfecta en el cariotipo de tetraploides de origen autoploide (duplicación de un genoma único) no contradice la condición de autoploidía y esperar una duplicación perfecta a partir del genoma diploide representa un caso ideal y poco frecuente en la

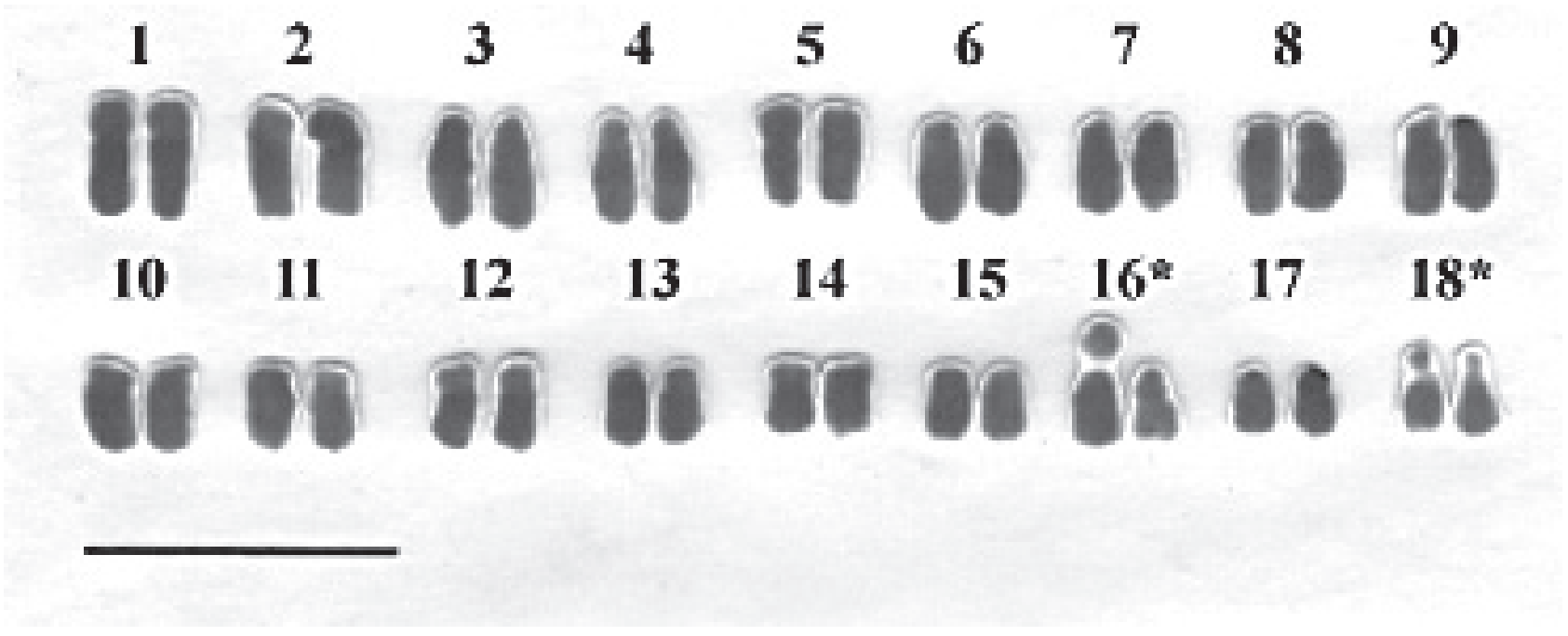

Figura 2. Cariotipo de Calochortus barbatus $(2 \mathrm{n}=4 \mathrm{x}=36)$. Los cromosomas están alineados por el centrómero y numerados en orden decreciente de tamaño. ${ }^{*}$ Cromosomas con satélite. Escala $=10 \mu \mathrm{m}$. 
naturaleza (Soltis et al., 2004; Font et al., 2008), siendo responsables de estas variaciones la acumulación gradual de diferencias estructurales en los cromosomas homólogos (dependiendo de la antigüedad del poliploide) y factores ecogeográficos que favorecen el surgimiento de ecotipos (Sybenga, 1969; Grant, 1981; Ramsey y Schemske, 2002).

Aunque el estudio citogenético por si solo no es suficiente para discernir el origen de un poliploide, la amplia distribución de $C$. barbatus en nuestro país favorece la idea de que se trata de una especie autoploide en la cual posibles eventos de hibridación intraespecífica (Soltis et al., 2003) ocurridos entre individuos diploides parcialmente aislados, han remodelado su cariotipo. Esto podría explicar no sólo las diferencias encontradas entre una simple duplicación de un genoma $2 x$ y el resultado obtenido aquí, sino la tolerancia ecológica de $C$. barbatus que sugiere las ventajas de un poliploide (Levin, 1983, 2002; Stebbins, 1985; Ramsey y Schemske, 1998; Soltis et al., 2003), así como el que sea considerada una especie variable (Ownbey, 1940; Gerritsen y Parsons, 2007).

Finalmente, ya que en el género Calochortus la asignación de especies a las secciones se ha basado en el número y morfología cromosómica, es necesaria una evaluación citogenética de las especies que habitan en nuestro país a fin de alcanzar una interpretación integral de su evolución, considerando además que han sido publicados análisis morfológicos y moleculares que muestran que la sección Cyclobothra es polifilética y que las relaciones filogenéticas entre varias de sus especies, incluida C. barbatus, no están resueltas (Ness, 1989; Patterson y Givnish, 2003).

\section{Agradecimientos}

Al Dr. Alfonso Delgado Salinas (Instituto de Biología, UNAM) la revisión crítica del manuscrito y sus valiosos comentarios. Las sugerencias de los revisores anónimos mejoraron sensiblemente la versión final.

\section{Literatura citada}

Beal, J. M. 1939. Cytological studies in relation to the classification of the genus Calochortus. Botanical Gazette 100:528-547.

Beal, J. M. 1941. Cytological studies in relation to the classification of the genus Calochortus. II. Botanical Gazette 102:810-811.

Beal, J. M. y M. Ownbey. 1943. Cytological studies in relation to the classification of the genus Calochortus. III. Botanical Gazette 104:553-562.
Cave, M. S. 1970. Chromosomes of the California Liliaceae. University of California Publications in Botany 57:1-58.

Font, M., J. Valles, A. Susana y N. Garcia-Jacas. 2008. Autoand allopolyploidy in Centaurea sect. Acrocentron s. $l$. (Asteraceae, Cardueae): karyotype and fluorochrome banding pattern analyses. Collectanea Botanica (Barcelona) 27:7-18.

Fredricks, N. A. 1989. Morphological comparison of Calochortus howellii and new species from southwestern Oregon, $C$. umpquaensis (Liliaceae). Systematic Botany 14:7-15.

Gerritsen, M. E. y R. Parsons. 2007. Calochortus: Mariposa lilies and their relatives. Timber, Portland, Oregon. 232 p.

Grant, V. 1981. Plant speciation. Columbia University Press, New York.

Levan, A., K. Fredga y A. A. Sandberg. 1964. Nomenclature for centromeric position on chromosomes. Hereditas 52:201219.

Levin, D. A. 1983. Polyploidy and novelty in flowering plants. American. Naturalist 122:1-25.

Levin, D. A. 2002. The role of chromosomal change in plant evolution. Oxford University Press, Oxford. 230 p.

McVaugh, R. 1989. Bromeliaceae to Dioscoreaceae. In Flora Novo-Galiciana. A descriptive account of the vascular plants of western México, vol. 15, W.R. Anderson (ed.). The University of Michigan Press, Ann Arbor. 398 p.

Martínez, M. 1979. Catálogo de nombres vulgares y científicos de plantas mexicanas. Fondo de Cultura Económica, México, D.F. 1247 p.

Nesom, G. L. 1983. New species of Calochortus (Liliaceae) and Linum (Linaceae) from northern México. Madroño 30:250254.

Ness, B. D. 1989. Seed morphology and taxonomic relationships in Calochortus (Liliaceae). Systematic Botany 14:495-505.

Newton, W. C. F. 1926. Chromosome studies in Tulipa and some related species. Journal of the Linnean Society (Botany) 47:339-354.

Ownbey, M. 1940. A monograph of the genus Calochortus. Annals of the Missouri Botanical Garden 27:371-560.

Patterson, T. B. y T. J. Givnish. 2003. Geographic cohesion, chromosomal evolution, parallel adaptive radiations, and consequent floral adaptations in Calochortus (Calochortaceae): evidence from a cpDNA phylogeny. New Phytologist 161:253-264.

Ramsey, J. y D. W. Schemske. 1998. Pathways, mechanisms and rates of polyploid formation in flowering plants. Annual Review of Ecology and Systematics 29:467-501.

Ramsey, J. y D. W. Schemske. 2002. Neopolyploidy in flowering plants. Annual Review of Ecology and Systematics 33:589-639.

Rzedowski, G. C. y J. Rzedowski. 2001. Flora fanerogámica del Valle de México. 2a ed., Instituto de Ecología y Comisión Nacional para el Conocimiento y Uso de la Biodiversidad, Pátzcuaro, Michoacán. 1406 p. 
Sinha, S. S. N. y H. Roy. 1979. Cytological studies in the genus Phaseolus I. Mitotic analysis in fourteen species. Cytologia 44:191-199.

Soltis, D. E., P. S. Soltis y J. A. Tate. 2003. Advances in the study of polyploidy since Plant Speciation. New Phytologist 161:173-191.

Soltis, D. E., P. S. Soltis, J. C. Pires, A. Kovarik, J. A. Tate y E. Mavrodiev. 2004. Recent and recurrent polyploidy in Tragopogon (Asteraceae): cytogenetic, genomic and genetic comparisons. Biological Journal of the Linnean Society 82:485-501.

Stebbins, G. L. 1985. Polyploid, hibridization, and the invasion of new habitats. Annals of the Missouri
Botanical Garden 72: 824-832.

Sybenga, J. 1969. Allopolyploidization of autopolyploids. I. Possibilities and limitations. Euphytica 18:355-371.

Tapia-Pastrana, F y P. Mercado-Ruaro. 2001. A combination of the "squash" and "splash" techniques to obtain the karyotype and assess meiotic behavior of Prosopis laevigata (Fabaceae: Mimosoideae). Cytologia 66:11-17.

Tapia-Pastrana, F., E. Gallegos-Pacheco, C. de Teodoro-Pardo y P. Mercado-Ruaro. 2005. New cytogenetic information of two Mexican populations of Crotalaria incana L. (LeguminosaePapilionoideae). Cytologia 70:207-212.

Tapia-Pastrana, F. 2007. Citogenética de Guazuma ulmifolia var. ulmifolia (Sterculiaceae). Darwiniana 45:23-27. 